\title{
Voice Analysis of the "White Peony Tune" of Linxia Huar Based on Acoustics
}

\author{
Yanbing Ding \\ Northwest University for Nationalities
}

\begin{abstract}
The paper collected the speech signal of Linxia Huar "White peony tune" which contain mountain singing, cheerful singing and tearful singing with experimental phonetic analysis method. And complete the signal tagging and parameter extraction. In the course of the study, the fundamental frequency and energy parameters was usesd to analysis the Basic phonetic features and prosodic features of Linxia Huar to explore the principle of its pronunciation and singing skills.
\end{abstract}

Keywords-Linxia Huar; white peony tune; phonetic features

\section{INTRODUCTION}

The Huar is only a kind of folk songs with love songs as its core which be widely sung commonly used by Chinese by minorities of Han ,Hui, Dongxiang and Baoan, salar, Tu, Yugur and Tibetan which have native language in the northwest region, mainly contain the connected area of Gansu, Qinghai, Ningxia, Xinjiang. The Huar were singing widely and has a long history, rich content and reputation at home and abroad. The Huar are the most loved by the people of the northwest, the girls called the beloved as "young", the young man called the beautiful girl as "flowers" [2] . In 2009, the "Gansu Huar was joint declarated by Gansu and other places which was on the list of the world's intangible cultural heritage Under the United Nations Educational, scientific and Cultural Organization. Its academic value also acquired more and more attention of the academic community.

The Huar are the most important folk oral tradition in Linxia, which carries some special spiritual complex and spiritual feelings of Linxia. With the progress of science and technology, especially the development of information technology,the protection and inheritance of the sound language and oral culture has better conditions. The methods of protecting the sound language and oral culture mainly used the king of text, voice and video at home. 1) The content of Huar mainly focuses on the music, rhythm and performance style research, and the discussion of the northwest culture, singing art and school. However, the Huar research from the view of acoustic and physiological point, with the modern voice equipment is seldom; 2) In the method, the existing method can record the related oral culture, but only part of the information, many rich and varied sound language and oral culture were difficult to completely preserve and inherit, so it is very important to study the Huar with the method of experimental phonetics technology to extract and keep its unique acoustics. In this paper, the "White peony tune" in Linxia is chosen to analysis the speech parameters, in order to reveal the intrinsic acoustic characteristics.

\section{EXPERIMENTAL METHOD AND EXPERIMENTAL ANALYSIS}

\section{A. Experimental Methods}

In the recording process of "Huar" of Linxia, the choice of informant plays a basic role for the pronunciation study, so the requirements for informant in the collection of data are very high. We have chosen a Linxia " Huar " singer who are more familiar with classic folk songs of Linxia " Huar "and have a profound understanding of the process of historical development of Linxia " Huar "as informant, and record his speech signa.

\section{B. Signal Acquisition}

The recording equipment contain electroglottography, external sound card, microphone and computer etc.The recording software using Audition 1.5. Acoustic analysis software using Praat, The acoustic parameters of the fundamental frequency and energy were extracted to analysis the music system of the Linxia " Huar ". The fundamental frequency react the speed of vocal fold vibration and the degree of control of the air flow rate. The energy react the level of volume, and the amplitude of the vibration in the process of singing [4].

\section{Acoustic Basis Analysis}

Linxia is known as "the hometown of Chinese Huar ", in October 19, 2004, the Chinese folk literature and Art Association awarded the title of hometown of Huar in Linxia, Gansu Province, Hui Autonomous prefecture. " Huar "are popular in Gansu Province, Linxia, Gannan, Minxian and other places with the unique style of folk songs, which with resounding loud and clear, bright, beautiful and agitation characteristics."White peony tune "is a king of tune named after the plant flowers, which has the short melody and the rhythm is more compact. Different people sing in different emotional state of the Huar have different flavors, so the three ways of singing of the white peony tune were showed one by one. The white peony tune has short text, In this paper, the acoustic analysis is mainly based on the fundamental frequency and energy parameters of the whole folk song.

1) Fundamental frequency analysis: The fundamental frequency represents the speed of vocal fold vibration and the control process of the air flow rate in the process of singing [4]. "White peony tune" vast mountain singing has free rhythm, melody, melodious high pitched vast, the frequency 
distribution diagram of the vast mountain singing chapter is shown in Figure I. "White peony tune" super singing has melody light singing, compact rhythm, frequency distribution of chapter of singing cry is shown in Figure II. White peony tune "crying singing has short rhythm, big fluctuation, the frequency distribution diagram of chapter of singing cry is shown in Figure III.

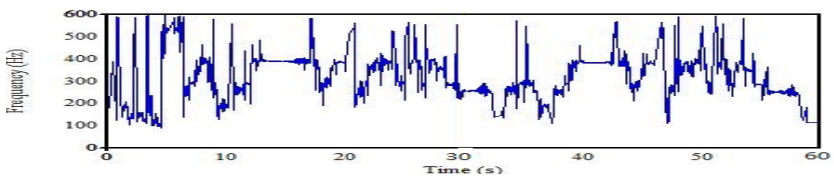

FIGURE I. "WHITE PEONY" WILD SINGING THE WHOLE VAST FUNDAMENTAL MAP

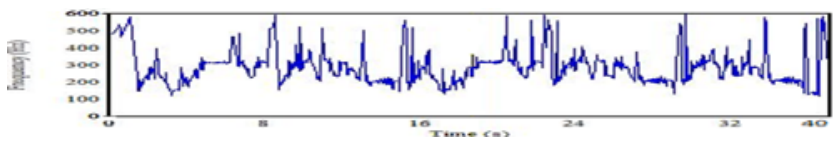

FIGURE II. "WHITE PEONY" SUPER HAPPY SINGING THE WHOLE FREQUENCY DIAGRAM

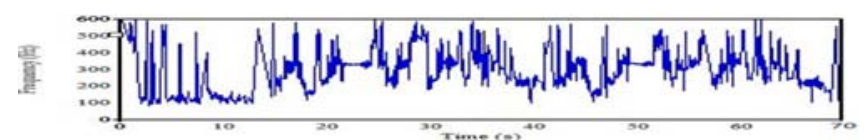

FIGURE III. "WHITE PEONY" CRY SINGING OVERALL FREQUENCY CHART

Can be seen from Figure I: "White peony tune" wild singing the whole vast frequency maximum value is $518.2 \mathrm{~Hz}$, the minimum value is $89.3 \mathrm{~Hz}$, the average value is $310.0 \mathrm{~Hz}$. The fundamental frequency of the whole song in a certain time range, always maintain a certain balance and stability, the formation of a number of range, volatility is relatively small, in the rhythm and emotional changes in the process of transformation, will suddenly increased or decreased. This shows that the singers singing in the process, the clever use of the soft palate arch, help to stop the subglottic vocal tension excess pressure air flow, accelerate the vocal cord vibration frequency, but energy is often maintained or changed very little, so long singers rely mainly on the smart control tone vibration frequency to enhance the sense of hearing effect. In addition, the singer also uses falsetto singing, increased range, to achieve a natural and friendly style, close to the spoken language, but without losing a strong penetration.

Can be seen from Figure II: "White peony tune" super happy singing the whole frequency maximum value is $499.2 \mathrm{~Hz}$, the minimum value is $116.5 \mathrm{~Hz}$, the average value is $268.3 \mathrm{~Hz}$. The song of the fundamental similarities and the vast mountain singing, in a certain time range, always maintain a certain balance and stability, the formation of a number of range, volatility is relatively small, but the cheerful singing super long than the vast mountain singing is much shorter, super happy singing rhythm relatively compact, the length of short.

Can be seen from Figure III: "White peony tune" cry singing the whole frequency maximum value is $522.6 \mathrm{~Hz}$, the minimum value is $83.8 \mathrm{~Hz}$, the average value is $271.5 \mathrm{~Hz}$. Tunes breathing position is very high, its basic suction location in the throat over the place, so the larynx closed tight shape, its sound is also on the inhale and cry at the same time, the sound of which was also shorter, no breath control.

Different singers have different treatment on "White peony tune" singing, and it's a different feeling of the music and performance for everybody, Coupled with the influence of singing emotion, which make the "white peony tune" singing vivid and distinctive.

2) Energy analysis: Energy represents the volume level, on behalf of the singer singing in the process of gas flow and the vibration amplitude of the level tone, Figure IV, V, VI for the "white peony tune" singing three chapter of the energy of the whole distribution map.

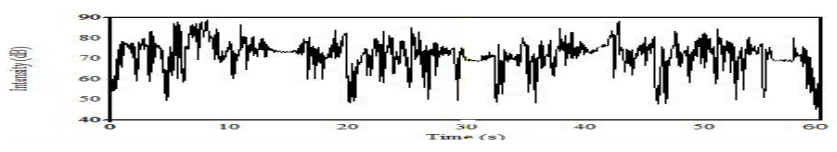

FIGURE IV. "WHITE PEONY" VAST MOUNTAIN SINGING OVERALL ENERGY MAP

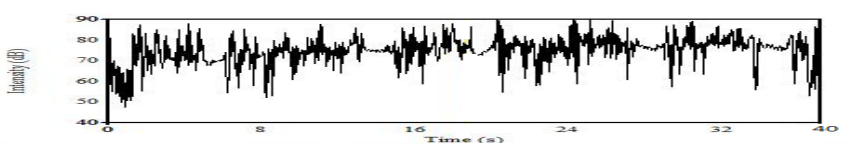

FIGURE V. "WHITE PEONY" SUPER SINGING CHEERFUL OVERALL ENERGY

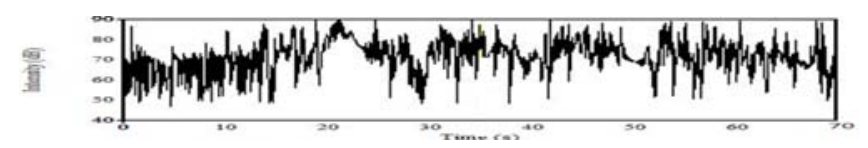

FIGURE VI. "WHITE PEONY" CRY SINGING OVERALL ENERGY MAP

Can be seen from Figure IV, V, VI:

"White peony tune" vast mountain singing overall energy maximum value is $90 \mathrm{~dB}$, the minimum value is $52.5 \mathrm{~dB}$, the average value is $77.42 \mathrm{~dB}$. "White peony tune" super singing cheerful overall energy maximum value is $83.07 \mathrm{~dB}$, the minimum value is $48.4 \mathrm{~dB}$, the average value is $60.42 \mathrm{~dB}$. "White peony tune" cry singing overall energy maximum value is $89.7 \mathrm{~dB}$, the minimum value is $49.6 \mathrm{~dB}$, the average value is $62.48 \mathrm{~dB}$. Three contrast, the vast mountain singing energy was higher than that of the cheerful singing and singing cheerful singing cry, ultra low energy, stable, energy fluctuation of singing and crying. Each singing to show energy "arch" trend, characteristics of the rise and fall is very consistent, compared to the general national singing and bel canto, "Huar" energy is not very high, but very concentrated, fluctuation is not obvious.

From point of view of the control and use of the breath, " Huar " singing art attach much weight to the respiratory training which plays a vital role in the singer singing, sound technology,. In the process of singing, the singer should have " Huar " by the vocal and vocal parts from deep down to breath on the formation of action and reaction, the ability to send special quality color pitch, and in this capacity to establish a sound and trigger a fulcrum, pleural cavity, oropharynx and hypopharynx overall, after the nasopharyngeal cavity, nasopharynx pipeline resonance front cavity, the head cavity 
resonance to provide the necessary possibility, so that the sound can have endurance and penetration. In different rhythm jump process, the uniform stability and continuity of energy need sound physiological mechanism and strong singing skills as a precondition[1].

\section{Singing Resonance Peak}

Other features of folk songs singing, singing in Linxia "Huar" also behaved most incisive, mainly reflected in the singing formant and vibrato, the singing breath control and respiratory rhythm has a very high demand. From the resonance characteristics of view, resonance in the singing sound is very important factor, is also an important means of expression in the art of singing, it can make a person's voice become clear, bright, more penetrating power. Singing "Huar ", in particular the need for such a bright, brilliant, penetrating power, metal like sound, only the sound, in the northwest to upload to send farther. Bel canto art also pay attention to the overall sound resonance.

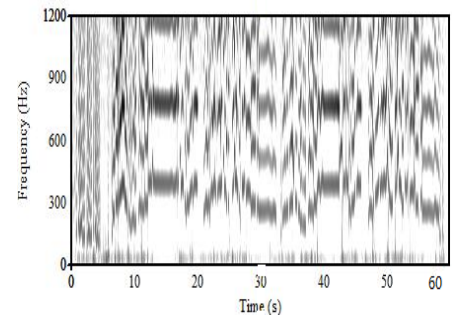

FIGURE VII. "WHITE PEONY" VAST MOUNTAIN SINGING IN FRENCH SPECTRUM

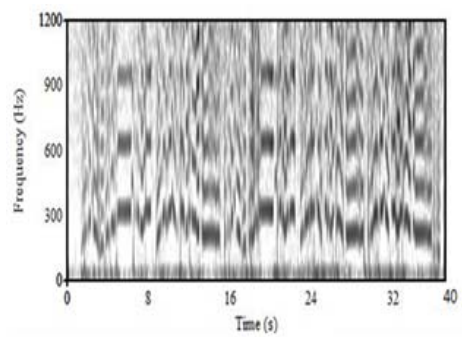

FIGURE VIII. "WHITE PEONY ORDER" SUPER HAPPY TO SING THE FRENCH SPECTRUM

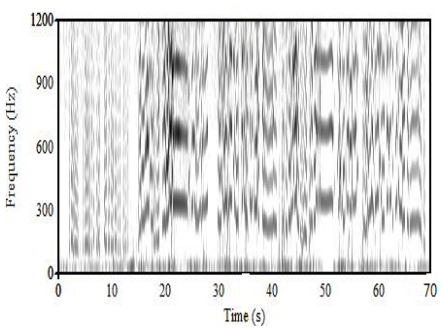

FIGURE IX. "WHITE PEONY ORDER" CRY CAVITY TO SING FRENCH SPECTRUM

Figure VII, VIII, IX for the extracted three kinds of singing of 3D graph. It can be seen from the figure, the vast mountain singing resonance peak Figure I is mainly in $350 \mathrm{~Hz}$, Figure II in $882 \mathrm{~Hz}$, a very Figure III, Figure IV and many other high frequency resonance produced by summit penetrating and loud at about $1200 \mathrm{~Hz} \sim 3000 \mathrm{~Hz}$ frequency near to $3000 \mathrm{~Hz}$ as the center of the high frequency resonance peak. Super happy singing formant Figure I mainly in $320 \mathrm{~Hz}$, Figure II in $740 \mathrm{~Hz}$. Cry singing formant Figure I mainly in $300 \mathrm{~Hz}$, Figure II in $700 \mathrm{~Hz}$. Because the singer throat drops, expanding the pharyngeal cavity, elongated channel, must strengthen the low-frequency resonance, and abundant bass is the basic condition to obtain high frequency overtone luster gorgeous, this is the " Huar " why not only the main reason of abundant high frequency, low frequency is also very full, but also have the reason of penetrating voice where. From the resonance characteristics of view, resonance in the singing sound is very important factor, is also an important means of expression in the art of singing, it can make a person's voice become clear, bright, more penetrating power. In addition, due to the " Huar " in the upper part of the singing art of resonance, will have a clear and accurate pronunciation; sound bright and clear, high concentration; the more flexible features, the artistic skills are different with Bel canto.

\section{CONCLUSION}

"Huar" is not only a kind of music form, is also a kind of Northwest spirit, which were likened to the hearts of the "beauty" of the songs by people in the West. This paper selected the representative the three kinds of singing of the white peony tune in Linxia area, analyzed the phonetic features of acoustic physiology from two levels of voice acoustic which contain the basic acoustic features (frequency and energy) and singing characteristics (singing formant) and draw some conclusions. Today, the people who sang "Huar "are rising, but the people who can fully control the style, content, skills, methods and the atmosphere of the release in singing of the Huar are seldom[2].The methods which only depend on the former "oral communication teaching" completely restricted the popularization and development and Inheritance of the " Huar " to a certain extent. The density alternate rhythms, a variety of acute accent singing, pale tone singing of the " Huar " are worthy of further study, how to protect the continuation of Linxia "Huar " and explore the singing essence is an urgent problem to be solved.

\section{ACKNOWLEDGMENT}

In the process of writing this paper, I have received many valuable suggestions from teachers, and also get the support and help from many students. Thank you for your concern, support and help to my teachers and students. In the end, this paper is the Nation Science Foundation Project (61462075).

\section{REFERENCES}

[1] Ladefoged, Peter1982. A course in Phonetics. 101-223.

[2] Zhao zongfu, The general theory of Huar, 1989, 1-26.

[3] Wu zongji, Lin maocan. Overview of experimental phonetics, 2014, 9(2), 301-379. 